\title{
Cardiac Arrest Due to Severe Dynamic Left Ventricular Outflow Obstruction and Hypertrophy Following Anesthesia Induction
}

\author{
Michael F Harrison ${ }^{1,2}$, Neil G Feinglass ${ }^{3}$, Emir Festic ${ }^{1, *}$
}

${ }^{1}$ Department of Critical Care Medicine, Mayo Clinic, Jacksonville, FL, USA

${ }^{2}$ Department of Emergency Medicine, Mayo Clinic, Jacksonville, FL, USA

${ }^{3}$ Department of Anesthesiology and Perioperative Medicine, Jacksonville, $\mathrm{FL}$, USA

\section{*Correspondence}

Festic.Emir@mayo.edu

(Emir Festic)

\begin{abstract}
Cardiac arrest following induction of general anesthesia is a rare event. A 47-yearold woman with a history of chronic neck pain secondary to spinal stenosis presented for elective cervical laminectomy. Induction of general anesthesia induced cardiac arrest and emergency insertion of the transesophageal echocardiogram probe identified severe, undiagnosed left ventricular hypertrophy with dynamic outflow obstruction. Resuscitative treatment was immediately implemented to include aggressive intravenous fluid resuscitation, intravenous esmolol and phenylephrine to augment preload, afterload, and reflex bradycardia effect. Return of spontaneous circulation was achieved and the patient was admitted to the ICU, where she was extubated with preserved neurocognitive function on the same day. Our case describes the risk presented by undiagnosed cardiac abnormalities in what was accepted as a low-to-intermediate risk patient undergoing an elective procedure. The increasing popularity and use of pocketsized handheld ultrasound devices may help reduce the risk of occurrences such as this in the future.
\end{abstract}

\section{Keywords}

Cardiac arrest, Cardiomyopathy, Hypertrophic, Echocardiogram, Transesophageal

\section{Introduction}

Cardiac arrest following induction of general anesthesia is a rare event that is often associated with the acuity of the situation, the patient's comorbidities, and the specific nature of the surgical intervention $[1,2]$. In addition, the patient is usually very well known to the medical staff present and thus treatment can be tailored to the precipitating cause(s) of is most often a witnessed cardiac arrest [3]. As a result, resuscitation and neurological outcomes are generally higher in perioperative cardiac arrest cases as compared to cases of cardiac arrest in the community or other in-hospital settings [3]. While laminectomy is an intermediate risk procedure, cervical procedures are low risk with respect to adverse cardiopulmonary events [4]. We present a case of cardiac arrest due to an undiagnosed comorbidity following induction of general anesthesia for an elective procedure. The patient was not contacted directly to review the manuscript; however, the patient provided a signed informed consent document at the time of admission that authorizes Mayo Clinic to disseminate and publish de-identified data in the form of research and/or academic publications if needed.

\section{Case History}

A 47-year-old woman with medical history significant only for chronic neck pain secondary to spinal stenosis presented to the outpatient surgical center for elective cervical laminectomy. Immediately following induction of general anesthesia with standard doses of propofol, rocuronium, and fentanyl the patient experienced cardiovascular collapse followed by cardiac arrest. No evidence of anaphylaxis or allergic drug reaction (bronchospasm, rash, flushing) could be identified. The presenting rhythms were sinus bradycardia followed by pulseless electrical activity (PEA). Treatment following the ACLS algorithm was initiated, including atropine during the peri-arrest episode of bradycardia followed by CPR and epinephrine during cardiac arrest. After initial lack of success in resuscitation efforts, the transesophageal echocardiogram (TEE 5Mhz) probe was inserted emergently and images of the left ventricle was obtained (Fig. 1). The prompt diagnosis of severe left ventricular hypertrophy and severe dynamic outflow obstruction (LVH) (ICD-9-CM 425.1, based on events occurring prior to 2015) with sudden cardiac arrest (ICD-9CM 427.5) due to hypovolemic shock (ICD-9-CM 276.52) was made and treatment was immediately adjusted to include aggressive intravenous fluid resuscitation, intravenous esmolol and phenylephrine to augment preload, afterload, and reflex bradycardia effect. Return of spontaneous circulation was 


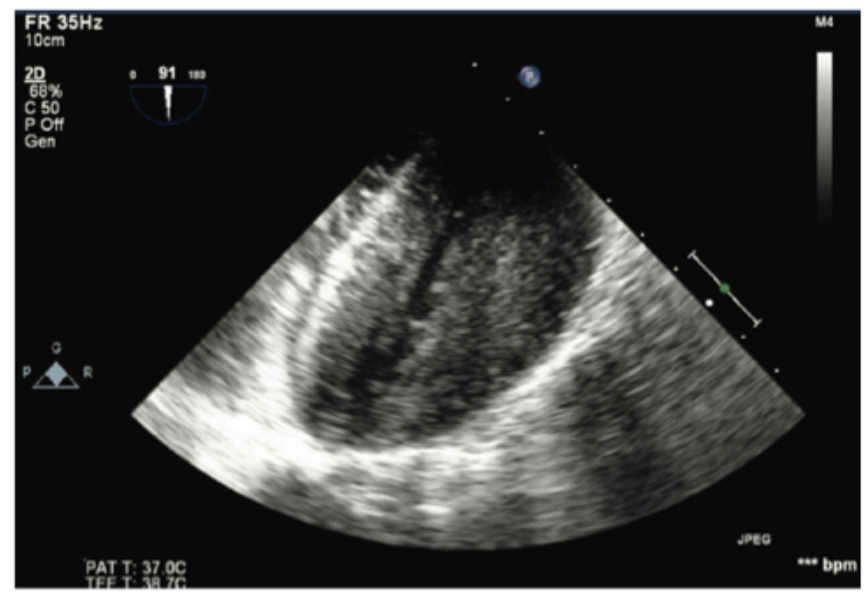

F IGURE 1. Emergency TEE image of left ventricle during resuscitation demonstrating almost complete cavity obliteration and severe LVH throughout the ventricle.

achieved and the patient was admitted to the ICU, where she was extubated with preserved neurocognitive function on the same day. Cardiology was consulted and the patient was discharged home to continue outpatient evaluation and management of LVH prior to rescheduling her surgery.

\section{Discussion}

Cardiac arrest following anesthesia is an extremely rare event $[1,2]$ but does present some unique differences as compared to cardiac arrest in or out of hospital. Specifically, cardiac arrest in the operating room most often occurs in a witnessed fashion in a patient whose medical history is well known to the practitioners present who, in the case of anesthesiologists, are experts in life support and resuscitation [3]. Prompt lifesaving intervention can begin immediately with targeted interventions that, for the reasons identified above, often do not follow the broad algorithms of advanced cardiac life support (ACLS) protocols. In fact, initial treatment based upon the ACLS algorithm to treat bradycardia (e.g. atropine) was not successful in stabilizing the patient and cardiac arrest ensued. However, tailored treatment subsequently occurred in this case with the administration of an alpha-agonist (phenylephrine) and a betaantagonist (esmolol), two medicines that are not recommended in the ACLS algorithm for cardiac arrest. Consistent with reports of increased rates of survival and favorable neurological outcomes following resuscitation [3], the patient in our case experienced no long term sequelae.

This event was surprising because increased rates of adverse cardiopulmonary events including cardiac arrest are not associated with cervical laminectomy surgery [4]. Emergent surgical cases involving elderly [1] or pediatric [2] patients represent the highest risk categories for cardiac arrest due to induction of anesthesia and the majority events are attributed to overdoses of anesthetic agents. Our patient did not fit the high-risk profile and her anesthesia dosing was appropriate. Specifically, her anesthesia induction included routine agents and intubation was performed successfully on first attempt without difficulty. Adequate ventilation and oxygenation were performed as per protocol and without difficulty even with the onset of bradycardia. Given the patient's rapid response to therapy directed specifically at augmenting her preload, afterload, and hypovolemic status, her undiagnosed cardiac disease is likely the precipitating cause. Perhaps even more surprising, her severe LVH and associated outflow obstruction was asymptomatic and undiagnosed until this event. Hypovolemia due to pre-operative nil per os (NPO) status and exacerbated by vasodilatation following general anesthesia induction is likely to have been an additional significant contributing factor in the events that transpired. However, based upon her age, her lack of known comorbidities, and the nature of the surgical intervention, our patient satisfied the criteria for low-to-intermediate risk and did not warrant further preoperative evaluation (i.e. electrocardiogram, echocardiogram, stress test) [4-6]. However, middle-aged women are underrepresented in these studies and even more so when the absence of known comorbidity is factored in to the risk stratification equation [7].

With respect to the patient's underlying $\mathrm{LVH}$, this condition had not been diagnosed prior to the performance of the TEE. Auscultation can distinguish the murmur associated with LVH from other murmurs, including innocent flow murmurs, approximately two-thirds of the time [8]. The pitfalls of relying upon auscultation alone to make the diagnosis LVH have been described [9] and it is likely that the impact of these pitfalls has only increased in the interim. The advent of pocketsized handheld point-of-care ultrasound (POCUS) devices and their increasing prevalence may provide a means by which to prevent situations such as described in this case in the future. These ultrasound devices and their ease of use can significantly increase the accuracy of in diagnosing cardiac abnormalities including LVH [10,11]. Scans, including cardiac studies, obtained with POCUS devices can provide clinically useful information in up to $95 \%$ cases [12]; furthermore, novice users such as internal medicine and anesthesiology residents in their first year of training are able to obtain "gold standard" cardiac images (96\% agreement with images obtained by cardiology specialists with specificity 0.91 , sensitivity 0.97 ) [13]. More than $85 \%$ of novice POCUS users are able to obtain cardiac images in $<10 \mathrm{~s}$ after one day of training [14]. As the costs of pocket sized devices decrease (approximately \$2000 USD currently), they are being issued to new medical students at the start of medical school [15].

To our knowledge based upon a review of the available literature, a prescribed algorithm and standardized approach to cardiopulmonary resuscitation following cardiac arrest due to LVH has not been published. Patients with LVH are at an increased risk of death due to cardiac arrhythmias such as ventricular tachycardia and ventricular fibrillation as compared to individuals without LVH [16]. The standard treatment for these arrhythmias is synchronized cardioversion or defibrillation, respectively. The prompt diagnose of the cause of this patient's cardiovascular collapse in conjunction with an expert understanding of cardiovascular physiology led to the administering of the tailored treatment that allowed resuscitative efforts to be successful in this case. 


\section{Conclusion}

Significant LVH does increase the risk associated with surgical procedures but, with TEE to monitor for early signs of dynamic outflow obstruction, successful outcomes can be achieved $[17,18]$. The utilization of pocket-sized handheld ultrasound devices may help clinicians identify patients with undiagnosed cardiac conditions before unexpected emergency situations arise during elective surgeries. Fortunately, our patient's resuscitation was successful and she did not have long term sequelae associated with her cardiac arrest.

\section{ACKNOWLEDGMENTS}

The favorable outcome in this case would not have been possible without the strong teamwork of the staff present in the operating room and ICU on a daily basis. The authors wish to acknowledge institutional support from Mayo Clinic Jacksonville. The authors would also like to express gratitude to the peer reviewers and editors for their constructive guidance during the peer review process.

\section{CONFLICT OF INTEREST}

The image and content of this submission have not been previously published. The authors have no financial conflict of interest to disclose.

\section{REFERENCES}

[1] Biboulet P, Aubas P, Dubourdieu J, et al. Fatal and non fatal cardiac arrests related to anesthesia. Canadian journal of anaesthesia. 2001;48:326-332.

[2] Keenan RL, Boyan CP. Cardiac arrest due to anesthesia: a study of incidence and causes. Jama. 1985;253:2373-2377.

[3] Moitra VK, Einav S, Thies K-C, et al. Cardiac arrest in the operating room: resuscitation and management for the anesthesiologist: part 1 . Anesth Analg. 2018;126:876-888.

[4] Passias PG, Poorman GW, Delsole E, et al. Adverse Outcomes and Prediction of Cardiopulmonary Complications in Elective Spine Surgery. Global Spine J. 2018;8:218-223.

[5] Palda VA, Detsky AS. Perioperative assessment and management of risk from coronary artery disease. Annals of internal medicine. 1997;127:313328.
[6] Eagle KA, Brundage B, Chaitman B, et al. Guidelines for perioperative cardiovascular evaluation for noncardiac surgery. Circulation. 1996;93:1278-1317.

[7] Ford MK, Beattie WS, Wijeysundera DN. Systematic review: prediction of perioperative cardiac complications and mortality by the revised cardiac risk index. Annals of internal medicine. 2010;152:26-35.

[8] Lembo NJ, Dell'Italia LJ, Crawford MH, et al. Bedside diagnosis of systolic murmurs. New England Journal of Medicine. 1988;318:15721578 .

[9] Craige E. Should auscultation be rehabilitated? Mass Medical Soc. 1988.

[10] Panoulas VF, Daigeler A-L, Malaweera ASN, et al. Pocket-size handheld cardiac ultrasound as an adjunct to clinical examination in the hands of medical students and junior doctors. European Heart Journal Cardiovascular Imaging. 2012;14:323-330.

[11] Bornemann P, Barreto TW. Point-of-care ultrasonography in family medicine. Am Fam Physician. 2018;98:200-202.

[12] Stachura M, Landes M, Aklilu F, et al. Evaluation of a point-of-care ultrasound scan list in a resource-limited emergency centre in Addis Ababa Ethiopia. African Journal of Emergency Medicine. 2017;7:118123.

[13] Frederiksen CA, Juhl-Olsen P, Andersen NH, et al. Assessment of cardiac pathology by point-of-care ultrasonography performed by a novice examiner is comparable to the gold standard. Scandinavian journal of trauma, resuscitation and emergency medicine. 2013;21:1-8.

[14] Price S, Ilper H, Uddin S, et al. Peri-resuscitation echocardiography: training the novice practitioner. Resuscitation. 2010;81:1534-1539.

[15] Comstock J. UC Irvine Medical School gifts Butterfly handheld ultrasounds to its whole class of 2023. Mobi Health News [Internet]. 2019 August 26, 2020. Available from: https://www.mobihealthnews. $\mathrm{com} /$ news/north-america/uc-irvine-medical-schoolgifts-butterfly-handheld-ultrasounds-its-whole-class.

[16] Chatterjee S, Bavishi C, Sardar P, et al. Meta-analysis of left ventricular hypertrophy and sustained arrhythmias. The American journal of cardiology. 2014;114:1049-1052.

[17] Cywinski JB, Argalious M, Marks TN, et al. Dynamic left ventricular outflow tract obstruction in an orthotopic liver transplant recipient. Liver transplantation. 2005;11:692-695.

[18] Ferguson E, Paech M, Veltman M. Hypertrophic cardiomyopathy and caesarean section: intraoperative use of transthoracic echocardiography. International journal of obstetric anesthesia. 2006;15:311-316.

How to cite this article: Michael F Harrison, Neil G Feinglass, Emir Festic. Cardiac Arrest Due to Severe Dynamic Left Ventricular Outflow Obstruction and Hypertrophy Following Anesthesia Induction. Signa Vitae. 2020;16(2):207-209. doi:10.22514/sv.2020.16.0071. 